\title{
Cys611Ser mutation in RET proto-oncogene in a kindred with medullary thyroid carcinoma and Hirschsprung's disease
}

\author{
Mikiko Nishikawa ${ }^{1,2}$, Yoshiki Murakumo ${ }^{1}$, Tsuneo Imai ${ }^{2}$, Kumi Kawai $^{1}$, Masahiro Nagaya ${ }^{3}$, \\ Hiroomi Funahashi ${ }^{2}$, Akimasa Nakao ${ }^{2}$ and Masahide Takahashiं ${ }^{\star 1}$
}

\begin{abstract}
${ }^{1}$ Department of Pathology, Nagoya University Graduate School of Medicine, 65 Tsurumai-cho, Showa-ku, Nagoya 466-8550, Japan; ${ }^{2}$ Department of Surgery II, Nagoya University Graduate School of Medicine, 65 Tsurumai-cho, Showa-ku, Nagoya 466-8550, Japan; ${ }^{3}$ Department of Pediatric Surgery, the Central Hospital, Aichi Prefectural Colony, 713-8 Kamiya-cho, Kasugai, Japan
\end{abstract}

Germline mutations in the RET proto-oncogene are responsible for the development of human hereditary diseases, including multiple endocrine neoplasia (MEN) type $2 \mathrm{~A}$ and 2B, familial medullary thyroid carcinoma (FMTC), and Hirschsprung's disease (HSCR). It has been reported that some families developed both MEN 2A/FMTC and HSCR, in which a mutation in a cysteine residue at codon 609, 618, or 620 in the RET gene was present. Here we report a novel RET mutation detected in a Japanese family with medullary thyroid carcinoma and HSCR. A germline mutation in cysteine 611 of the RET gene was identified in this family, which introduced an amino-acid change from cysteine to serine. By biological and biochemical analyses of mutant RET proteins, we previously predicted the potentiality that amino-acid substitution for cysteine 611 as well as cysteines 609,618 , and 620 would promote the development of MEN 2A/FMTC and HSCR. This clinical case substantiates our suggestion for the mechanism of the development of both the diseases.

European Journal of Human Genetics (2003) 11, 364-368. doi:10.1038/sj.ejhg.5200971

Keywords: RET mutation; multiple endocrine neoplasia; medullary thyroid carcinoma; Hirschsprung's disease

\section{Introduction}

The RET proto-oncogene encodes a transmembrane receptor with a tyrosine kinase domain that is expressed in cells derived from neural crest. $^{1-3}$ It is known that its germline mutations develop several human hereditary disorders, including multiple endocrine neoplasia type 2 (MEN 2) and Hirschsprung's disease (HSCR)., ${ }^{4,5}$ MEN 2 is an autosomal dominant neoplastic syndrome that is classified into three clinical subtypes, MEN 2A, MEN 2B, and familial medullary thyroid carcinoma (FMTC). MEN 2A is character-

*Correspondence: Dr M Takahashi, Department of Pathology, Nagoya University Graduate School of Medicine, 65 Tsurumai-cho, Showa-ku, Nagoya 466-8550, Japan. Tel: +81 52744 2093; Fax: +81 52744 2098; E-mail: mtakaha@med.nagoya-u.ac.jp

Received 22 October 2002; revised 17 January 2003; accepted 24 January 2003 ized by the clinical feature of medullary thyroid carcinoma (MTC), pheochromocytoma, and parathyroid hyperplasia. MEN 2B patients are affected by a more complex phenotype including MTC, pheochromocytoma, skeletal abnormalities, and mucosal neuroma, whereas FMTC is defined by the sole phenotype of MTC without any other abnormalities. HSCR is a neurocristopathy characterized by the absence of intrinsic ganglion cells in the distal gastrointestinal tract, and is inherited in a non-Mendelian fashion. ${ }^{6}$

The mechanisms of development of MEN 2 and HSCR by RET mutations are quite different. MEN 2 and HSCR are caused by its 'gain of function' and 'loss of function' mutations, respectively. ${ }^{4}$ In MEN 2A patients, mutations are mostly detected in one of the six cysteine residues at codons $609,611,618,620,630$, and 634 in the RET extracellular cysteine-rich domain. These cysteine mutations 
constitutively activate RET by inducing its disulfide-linked homodimerization. $^{7-9}$ On the other hand, MEN $2 B$ mutations are detected at codon 883 or 918 in the RET intracellular kinase domain, leading to RET activation without dimer formation. ${ }^{9-11}$ In FMTC patients, most $R E T$ mutations are identified in cysteine residues like the MEN $2 A$ mutations, and some are at codon 768,804 , or 891 in the intracellular kinase domain. HSCR mutations including missense, nonsense, deletion, insertion, and frameshift mutations were reported throughout the whole coding region of RET gene without any hot spots, and impair RET function by various mechanisms. We and others demonstrated that HSCR mutations identified in the RET extracellular domain markedly impair the RET cell surface expression, whereas the mutations in the kinase domain impair the RET kinase activity or downstream signaling. ${ }^{12-16}$

Interestingly, the development of both MEN 2A/FMTC and HSCR has been reported in some families. ${ }^{17-29}$ The screening for germline mutations of the RET protooncogene revealed that a single amino-acid substitution in one of the three cysteine residues at codons 609, 618, and 620 is involved in the development of both diseases. In this study, we report the first clinical case to prove the pathogenesis of a cysteine 611 mutation for the development of both MTC and HSCR.

\section{Patients and methods Patients}

Figure 1 shows the pedigree of the family described in this paper. The proband (II-2), a 47-year-old male, underwent left hemithyroidectomy for preoperative diagnosis as papillary thyroid carcinoma of the left thyroid gland in Aichi Cancer Center Hospital (Nagoya, Japan). It was diagnosed as an MTC by the postoperative histopathological examination of his surgical specimens. His family history showed that the proband's father (I-1) died at the age of 56 years from an advanced MTC in Nagoya University Hospital (Nagoya, Japan). In addition, the proband's son (III-3) underwent an operation for HSCR (short segment type) at the age of 1 year in the Central Hospital, Aichi Prefectural Colony (Kasugai, Japan).

On the basis of these episodes, an inherited disorder was speculated in this family. The proband was admitted to our hospital for further examinations and treatments. The molecular examinations for germline RET mutations in the affected family members were performed under informed consent and permission of ethical committee of Nagoya University School of Medicine. The examination of the proband's father was performed by agreement from his family.

\section{DNA preparation}

Genomic DNA of the proband was extracted from peripheral blood leukocytes according to the standard protocols.

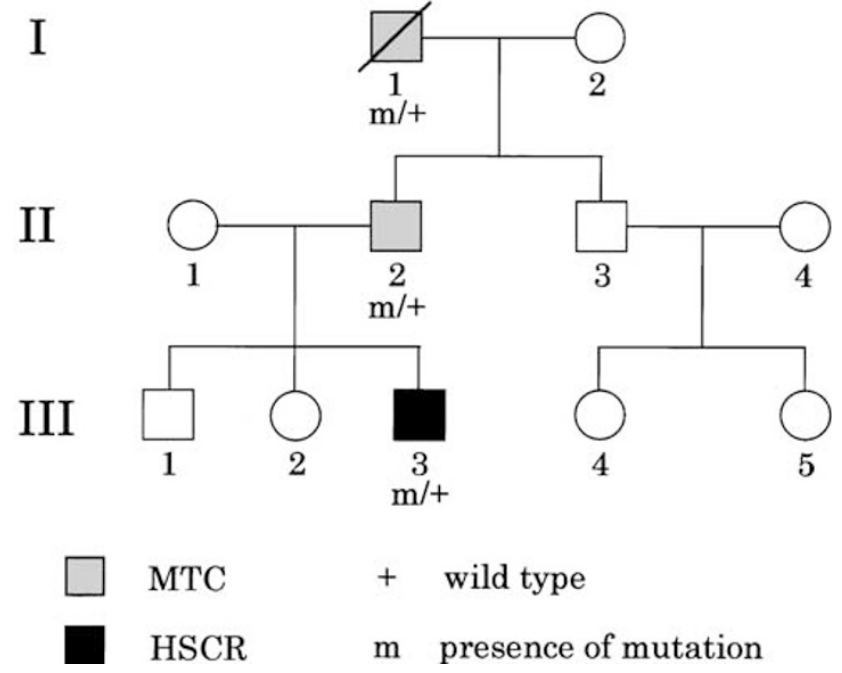

Figure 1 The pedigree of the family with MTC and HSCR. Individual II-2 is the proband.

Genomic DNA of the proband's son was extracted from the paraffin-embedded tissue sections of his ileum that was resected at the operation for adhesive ileus. Three pieces of 10- $\mu$ m-thick paraffin-embedded tissue sections were incubated in xylene for $30 \mathrm{~min}$ in an Eppendorf tube to remove paraffin from the tissues. Xylene was discarded, and the tissues were washed with ethanol and incubated in proteinase K buffer (10 mM Tris- $\mathrm{HCl}, \mathrm{pH} 8.3,2.5 \mathrm{mM} \mathrm{MgCl}_{2}$, $0.45 \%$ Tween $20,0.45 \% \mathrm{NP}-40,10 \mathrm{mg} / \mathrm{ml}$ proteinase $\mathrm{K}$ ) overnight. The DNAs were purified by phenol/chloroform DNA extraction method. Genomic DNA of the proband's father was also isolated from the paraffin-embedded tissue sections of his autopsied liver.

\section{Mutation analysis}

Sets of oligonucleotide primers to amplify the protein coding sequences of the RET gene were designed based on the published genomic sequences. ${ }^{30}$ PCR reactions were performed using the genomic DNAs of the proband, proband's son, and proband's father as templates, and the PCR products were subjected to sequencing directly, or they were cloned into pGEM-T plasmids (Promega, Madison, WI, USA) and sequenced. Double-strand sequencing of the PCR products and the inserts in pGEM-T plasmids was performed by a cycle sequencing program using the BigDye $^{\mathrm{TM}}$ Terminator Cycle Sequencing Kit (Applied Biosystems, Foster City, CA, USA). Nucleotide sequences were determined by an automated Applied Biosystems sequencer model 310 (Applied Biosystems, Foster City, CA, USA).

\section{Results}

A 47-year-old male (II-2 in Figure 1) underwent a completion thyroidectomy with bilateral lymph node dissection. 
Small lesions of the remnant thyroid were diagnosed as MTC, but no pathological changes such as adenoma or hyperplasia were detected in the biopsy specimens of the parathyroid glands. It was also pointed out that he had a small tumor $(2 \mathrm{~cm}$ in diameter) in the left adrenal gland by a screening computed tomography (CT) examination. His serum and urinary catecholamine levels and other adrenal hormones were within normal range, and he has not undergone an operation to resect the adrenal tumor to date. However, it seems likely that the left adrenal tumor is pheochromocytoma, thus, the possibility that he may be a MEN 2A patient is not excluded.

The sequences of RET exons 10 and 11, where mutations are usually present in MEN 2A/FMTC patients, were examined, and a TGC to TCT heterozygous germline mutation was detected in codon 611 of the RET gene, resulting in the amino-acid change from cysteine to serine (C611S) (Figure 2). The proband's son developed HSCR and was also screened for RET mutations. The same heterozygous mutation in codon 611 was detected. No other nucleotide alterations were found in the whole coding sequence of the RET gene of the proband's son. The same C611S mutation was present in the proband's father who died of MTC.

\section{Discussion}

A total of 22 families with RET mutations that developed both MEN 2A/FMTC and HSCR were previously reported. ${ }^{18-29}$ The RET mutations identified in these families include two C609Y, five C618R, three C618S, eight C620R, three C620S, and one C620W mutations. In the present report, we described a new family affected with both MTC and HSCR. The mutation analyses of the RET gene revealed that a substitution of serine for cysteine at codon 611 was detected in affected members of the family. This family did not fulfill the definition of MEN 2A or FMTC, because MTC developed in only two members so far. Despite this fact, this is the first case of a family that suggests the pathogenesity of RET codon 611 mutation for the development of MTC and HSCR that are caused by gain-of-function and loss-of-function mutations of RET, respectively.

We previously proposed a possible mechanism for the development of both diseases by a single cysteine mutation in $R E T^{4,31,32}$ In NIH3T3 cells, the transforming activities of the RET proteins harboring the mutations in cysteine 609, 611,618 , or 620 were significantly lower than those harboring the mutations in cysteine $634 .^{31,33}$ The low transforming activities of the former mutant proteins were caused by impairment of their cell surface expression. Cysteine 609, 611, 618 or 620 mutations may alter the correct folding of RET as observed with the HSCR mutations identified in the RET extracellular domain, ${ }^{13,14}$ thereby interfering with RET maturation, intracellular
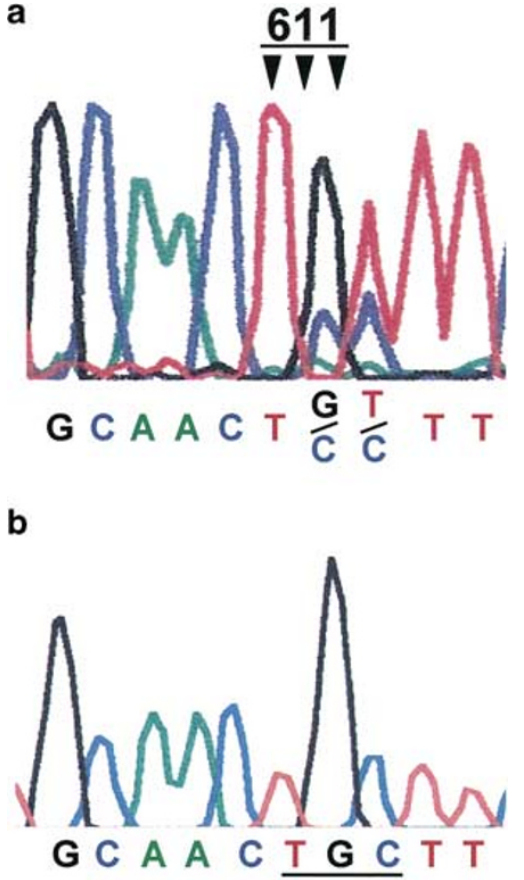

C

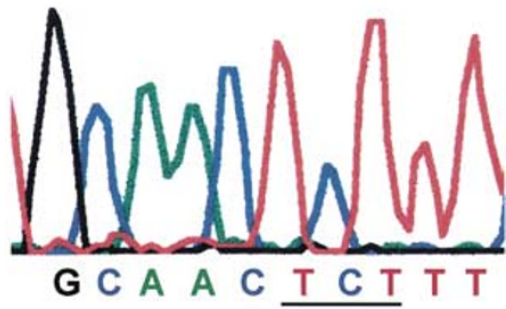

Figure 2 C611S mutation detected in the family. Sequences of $R E T$ exon 10 of the proband were determined by direct sequencing of the PCR products (a). RET exon 10 was also sequenced after subcloning of the PCR products into the pGEM-T plasmid (b, c). A TGC to TCT heterozygous mutation was detected at codon 611. (b) wild-type allele, (c) mutant allele.

trafficking or stable expression on the cell surface. The signal through the reduced RET proteins on the cell surface would not be sufficient for the differentiation of the intestinal ganglion cells, resulting in the development of HSCR.

In addition, cysteine $609,611,618$, or 620 mutations can induce ligand-independent RET dimerization at low levels responsible for the transforming activity in NIH3T3 cells. $^{31,33}$ Since these mutations were also identified in many MEN 2A/FMTC families, the low transforming activity appears to be sufficient to trigger the development of MTC. Based on these data, we predicted that cysteine 611 mutations have the potential to develop both HSCR and MTC. ${ }^{31}$ Mutation analysis of the family reported here supported our prediction. 
Another interesting point of the present case is that two nucleotides of RET codon 611 were substituted (from TGC to TCT). No case that shows two-nucleotide alteration in one codon of the RET gene was previously reported, although some cases that have two codons with onenucleotide alteration in the RET gene were reported. ${ }^{27}$ In addition, the present case showed no nucleotide alterations at polymorphic codons in the RET gene that may affect the development of HSCR. ${ }^{34-38}$

Germline mutations in several genes including GDNF, neurturin, endothelin receptor $\mathrm{B}$, endothelin-3, SOX10, endothelin-converting enzyme 1 , and ZFHX1B were reported in HSCR patients. However, the mutation frequencies of these genes in HSCR patients are significantly low compared with that of the RET gene. ${ }^{39-47}$ Furthermore, some other loci were proposed to be associated with the risk of HSCR. ${ }^{48}$ Although the possibility that the mutations of these genes are present and affect the development of HSCR in this case is not excluded, it is not practical to screen all the genes. Among the genes described above, the RET status is the most important for follow-up of HSCR patients because the patients who have a mutation at codon $609,611,618$, or 620 in the RET gene are at risk for the development of MTC. Thus, the screening for these codons in the RET gene should be performed in all cases of HSCR routinely.

\section{Acknowledgements}

We thank Mr Y Imaizumi, Mr K Uchiyama, and Miss M Kozuka for technical assistances. This work was supported by a grant-in-aid for center of excellence (COE) research from the Ministry of Education, Culture, Sports, Science and Technology of Japan.

\section{References}

1 Takahashi M, Buma Y, Iwamoto T, Inaguma Y, Ikeda H, Hiai H: Cloning and expression of the ret proto-oncogene encoding a tyrosine kinase with two potential transmembrane domains. Oncogene 1988; 3: 571-578.

2 Pachnis V, Mankoo B, Costantini F: Expression of the c-ret protooncogene during mouse embryogenesis. Development 1993; 119: 1005-1017.

3 Tsuzuki T, Takahashi M, Asai N, Iwashita T, Matsuyama M, Asai J: Spatial and temporal expression of the ret proto-oncogene product in embryonic, infant and adult rat tissues. Oncogene 1995; 10: 191-198.

4 Takahashi M: The GDNF/RET signaling pathway and human diseases. Cytokine Growth Factor Rev 2001; 12: 361-373.

5 Manié S, Santoro M, Fusco A, Billaud M: The RET receptor: function in development and dysfunction in congenital malformation. Trends Genet 2001; 17: 580-589.

6 Amiel J, Lyonnet S: Hirschsprung disease, associated syndromes, and genetics: a review. J Med Genet 2001; 38: 729-739.

7 Asai N, Iwashita T, Matsuyama M, Takahashi M: Mechanism of activation of the ret proto-oncogene by multiple endocrine neoplasia 2A mutations. Mol Cell Biol 1995; 15: 1613-1619.

8 Borrello MG, Smith DP, Pasini B et al: RET activation by germline MEN2A and MEN2B mutations. Oncogene 1995; 11: 2419-2427.
9 Santoro M, Carlomagno F, Romano A et al: Activation of RET as a dominant transforming gene by germline mutations of MEN2A and MEN2B. Science 1995; 267: 381-383.

10 Iwashita T, Asai N, Murakami H, Matsuyama M, Takahashi M: Identification of tyrosine residues that are essential for transforming activity of the ret proto-oncogene with MEN2A or MEN2B mutation. Oncogene 1996; 12: 481-487.

11 Iwashita T, Kato M, Murakami H et al: Biological and biochemical properties of Ret with kinase domain mutations identified in multiple endocrine neoplasia type $2 \mathrm{~B}$ and familial medullary thyroid carcinoma. Oncogene 1999; 18: 3919-3922.

12 Pasini B, Borrello MG, Greco A et al: Loss of function effect of RET mutations causing Hirschsprung disease. Nat Genet 1995; 10: 35-40.

13 Carlomagno F, De Vita G, Berlingieri MT et al: Molecular heterogeneity of RET loss of function in Hirschsprung's disease. EMBO J 1996; 15: 2717-2725.

14 Iwashita T, Murakami H, Asai N, Takahashi M: Mechanism of Ret dysfunction by Hirschsprung mutations affecting its extracellular domain. Hum Mol Genet 1996; 5: 1577-1580.

15 Iwashita T, Kurokawa K, Qiao S et al: Functional analysis of RET with Hirschsprung mutations affecting its kinase domain. Gastroenterology 2001; 121: 24-33.

16 Bordeaux MC, Forcet C, Granger L et al: The RET proto-oncogene induces apoptosis: a novel mechanism for Hirschsprung disease. EMBO J 2000; 19: 4056-4063.

17 Verdy M, Weber AM, Roy CC, Morin CL, Cadotte M, Brochu P: Hirschsprung's disease in a family with multiple endocrine neoplasia type 2. J Pediatr Gastroenterol Nutr 1982; 1: 603-607.

18 Chretien P, Blanchard L, Gaboury L, Lacroix A, Verdy M, Villeneuve L: Cosegregation of Hirschsprung's disease (HSCR) and a RET mutation in a French Canadian family with MEN 2A. Am J Hum Genet 1994; 55: A357.

19 Mulligan LM, Eng C, Attie T et al: Diverse phenotypes associated with exon 10 mutations of the RET proto-oncogene. Hum Mol Genet 1994; 3: 2163-2167.

20 Borst MJ, VanCamp JM, Peacock ML, Decker RA: Mutational analysis of multiple endocrine neoplasia type $2 \mathrm{~A}$ associated with Hirschsprung's disease. Surgery 1995; 117: 386-391.

21 Blank RD, Sklar CA, Dimich AB, LaQuaglia MP, Brennan MF: Clinical presentations and RET protooncogene mutations in seven multiple endocrine neoplasia type 2 kindreds. Cancer 1996; 78: 1996-2003.

22 Caron P, Attie T, David D et al: C618R mutation in exon 10 of the $R E T$ proto-oncogene in a kindred with multiple endocrine neoplasia type 2A and Hirschsprung's disease. J Clin Endocrinol Metab 1996; 81: 2731-2733.

23 Peretz H, Luboshitsky R, Baron E et al: Cys 618 Arg mutation in the $R E T$ proto-oncogene associated with familial medullary thyroid carcinoma and maternally transmitted Hirschsprung's disease suggesting a role for imprinting. Hum Mutat 1997; 10: 155-159.

24 Borrego S, Eng C, Sanchez B, Saez ME, Navarro E, Antinolo G: Molecular analysis of the ret and GDNF genes in a family with multiple endocrine neoplasia type $2 \mathrm{~A}$ and Hirschsprung disease. J Clin Endocrinol Metab 1998; 83: 3361-3364.

25 Decker RA, Peacock ML, Watson P: Hirschsprung disease in MEN 2A: increased spectrum of RET exon 10 genotypes and strong genotype-phenotype correlation. Hum Mol Genet 1998; 7: $129-134$

26 Romeo G, Ceccherini I, Celli J et al: Association of multiple endocrine neoplasia type 2 and Hirschsprung disease. J Intern Med 1998; 243: 515-520.

27 Sijmons RH, Hofstra RM, Wijburg FA et al: Oncological implications of RET gene mutations in Hirschsprung's disease. Gut 1998; 43: 542-547.

28 Inoue $\mathrm{K}$, Shimotake $\mathrm{T}$, Inoue $\mathrm{K}$, Tokiwa $\mathrm{K}$, Iwai N: Mutational analysis of the RET proto-oncogene in a kindred with multiple endocrine neoplasia type $2 \mathrm{~A}$ and Hirschsprung's disease. J Pediatr Surg 1999; 34: 1552-1554. 
29 Pasini B, Rossi R, Ambrosio MR et al: RET mutation profile and variable clinical manifestations in a family with multiple endocrine neoplasia type 2A and Hirschsprung's disease. Surgery 2002; 131: 373-381.

30 Ceccherini I, Hofstra RM, Luo Y et al: DNA polymorphisms and conditions for SSCP analysis of the 20 exons of the ret protooncogene. Oncogene 1994; 9: 3025-3029.

31 Ito S, Iwashita $\mathrm{T}$, Asai $\mathrm{N}$ et al: Biological properties of Ret with cysteine mutations correlate with multiple endocrine neoplasia type 2A, familial medullary thyroid carcinoma, and Hirsch-sprung's disease phenotype. Cancer Res 1997; 57: 2870-2872.

32 Takahashi M, Iwashita T, Santoro M, Lyonnet S, Lenoir GM, Billaud M: Co-segregation of MEN2 and Hirschsprung's disease: the same mutation of RET with both gain and loss-of-function?. Hum Mutat 1999; 13: 331-336.

33 Chappuis-Flament S, Pasini A, De Vita G et al: Dual effect on the RET receptor of MEN 2 mutations affecting specific extracytoplasmic cysteines. Oncogene 1998; 17: 2851-2861.

34 Fitze G, Schreiber M, Kuhlisch E, Schackert HK, Roesner D: Association of RET protooncogene codon 45 polymorphism with Hirschsprung disease. Am J Hum Genet 1999; 65: 1469-1473.

35 Fitze G, Cramer J, Ziegler A et al: Association between c135G/A genotype and RET proto-oncogene germline mutations and phenotype of Hirschsprung's disease. Lancet 2002; 359: 1200-1205.

36 Borrego S, Saez ME, Ruiz A et al: Specific polymorphisms in the RET proto-oncogene are over-represented in patients with Hirschsprung disease and may represent loci modifying phenotypic expression. J Med Genet 1999; 36: 771-774.

37 Borrego S, Ruiz A, Saez ME et al: RET genotypes comprising specific haplotypes of polymorphic variants predispose to isolated Hirschsprung disease. J Med Genet 2000; 37: 572-578.
38 Sancandi M, Ceccherini I, Costa M: Incidence of RET mutations in patients with Hirschsprung's disease. J Pediatr Surg 2000; 35: 139-142.

39 Puffenberger EG, Hosoda K, Washington SS et al: A missense mutation of the endothelin-B receptor gene in multigenic Hirschsprung's disease. Cell 1994; 79: 1257-1266.

40 Angrist M, Bolk S, Halushka M, Lapchak PA, Chakravarti A: Germline mutations in glial cell line-derived neurotrophic factor $(G D N F)$ and RET in a Hirschsprung disease patient. Nat Genet 1996; 14: 341-344.

41 Edery P, Attie T, Amiel J et al: Mutation of the endothelin-3 gene in the Waardenburg-Hirschsprung disease (Shah-Waardenburg syndrome). Nat Genet 1996; 12: 442-444.

42 Salomon R, Attie T, Pelet A et al: Germline mutations of the RET ligand GDNF are not sufficient to cause Hirschsprung disease. Nat Genet 1996; 14: 345-347.

43 Doray B, Salomon R, Amiel J et al: Mutation of the RET ligand, neurturin, supports multigenic inheritance in Hirschsprung disease. Hum Mol Genet 1998; 7: 1449-1452.

44 Pingault V, Bondurand N, Kuhlbrodt K et al: SOX10 mutations in patients with Waardenburg-Hirschsprung disease. Nat Genet 1998; 18: 171-173.

45 Hofstra RM, Valdenaire $\mathrm{O}$, Arch E et al: A loss-of-function mutation in the endothelin-converting enzyme 1 (ECE-1) associated with Hirschsprung disease, cardiac defects, and autonomic dysfunction. Am J Hum Genet 1999; 64: 304-308.

46 Wakamatsu N, Yamada Y, Yamada K et al: Mutations in SIP1, encoding Smad interacting protein-1, cause a form of Hirschsprung disease. Nat Genet 2001; 27: 369-370.

47 Nagaya M, Kato J, Niimi N, Tanaka S, Wakamatsu N: Clinical features of a form of Hirschsprung's disease caused by a novel genetic abnormality. J Pediatr Surg 2002; 37: 1117-1122.

48 Gabriel SB, Salomon R, Pelet A: Segregation at three loci explains familial and population risk in Hirschsprung disease. Nat Genet 2002; 31: 89-93. 